\title{
Interval Estimation in a Two Parameter Weibull Distribution Based on Type-2 Censored Data
}

\author{
Raphael Masila Mweleli, Luke Akong'o Orawo, Cox Lwaka Tamba, Justin Obwoge Okenye \\ Department of Mathematics, Egerton University, Egerton, Kenya \\ Email: raphaelrampz@yahoo.com,orawo2000@yahoo.com,clwaka@yahoo.com,justinoke69@gmail.com
}

How to cite this paper: Mweleli, R.M., Orawo, L.A., Tamba, C.L. and Okenye, J.O. (2020) Interval Estimation in a Two Parameter Weibull Distribution Based on Type-2 Censored Data. Open Journal of Statistics, 10, 1039-1056. https://doi.org/10.4236/ojs.2020.106059

Received: November 3, 2020

Accepted: December 18, 2020

Published: December 21, 2020

Copyright $\odot 2020$ by author(s) and Scientific Research Publishing Inc. This work is licensed under the Creative Commons Attribution International License (CC BY 4.0).

http://creativecommons.org/licenses/by/4.0/ (c) (i) Open Access

\begin{abstract}
In this paper, we consider the construction of the approximate profile- likelihood confidence intervals for parameters of the 2-parameter Weibull distribution based on small type- 2 censored samples. In previous research works, the traditional Wald method has been used to construct approximate confidence intervals for the 2-parameter Weibull distribution under type-2 censoring scheme. However, the Wald technique is based on normality assumption and thus may not produce accurate interval estimates for small samples. The profile-likelihood and Wald confidence intervals are constructed for the shape and scale parameters of the 2-parameter Weibull distribution based on simulated and real type- 2 censored data, and are hence compared using confidence length and coverage probability.
\end{abstract}

\section{Keywords}

Two-Parameter Weibull Distribution, Interval Estimation, Relative Likelihood Function, Maximum Relative Likelihood Function, Profile-Likelihood Interval, Coverage Probability

\section{Introduction}

On most occasions, when performing life testing experiments, the main interest is to examine the lifespan of a specimen. For instance, tests might be carried out to determine the lifespan of an aircraft wing prior to failure from metal fatigue. Such experiments are expensive and time-consuming, and only a few units can be inspected. Due to time and financial constraints, a researcher may not be able to examine the failure time of all the units under investigation [1] and [2]. One is prompted to set an appropriate censoring limit after which the experiment is terminated. The termination of observations in life test experiments due to 
causes other than the natural failure to which an item is subjected is referred to as censoring.

Type- 2 censoring arises when a set of identical items are subjected to a life test, and the experiment is stopped when a predetermined number of items are observed to have failed, and after that, the remaining items are right censored [3]. Type- 2 censoring scheme is one of the most popular censoring schemes used in reliability and life testing experiments. This is because most of the life testing experiments especially in the field of engineering and medicine require one to choose a sample size that will minimize costs.

Some of the common statistical distributions used to fit censored life data include Rayleigh [4], Gamma [5], Gumbel [6], logistic [7], lognormal [8], log- logistic [9], exponential [10], normal [11], Weibull [12] and [13], and Mixed Weibull [14].

From the aforementioned life distributions, Weibull distribution is the most preferred distribution since it can take characteristics of other life distributions based on the value of its shape parameter [15]. Although, there are different forms of Weibull distributions, 2-parameter Weibull distribution and 3-parameter Weibull distribution are the most commonly used forms in fitting censored life data.

Point estimation in a 2-parameter Weibull distribution or 3-parameter Weibull distribution in the presence of censored data can be done using various techniques. These methods include; maximum likelihood estimation (MLE) [16], a method of moments (MOM) [17], expectation maximization (EM) algorithm [13], and generalized least square (GLS) [18].

Interval estimation in a 2-parameter Weibull distribution based on large samples has been carried out in several ways; including Bayesian and Wald [19]. In previous research works, Wald method has been employed to construct approximate confidence intervals for the parameters of this distribution under type-2 censoring scheme [3]. However, this method is appropriate for large samples and may give inaccurate interval estimates when the sample size is small. Hence, there is a need to explore other methods of constructing confidence intervals, especially on small samples. The profile-likelihood technique comes in handy. This technique has not been used previously to construct approximate confidence intervals for parameters of 2-parameter Weibull distribution under small type- 2 censored samples.

The profile-likelihood method is used in situations where the focus is on a subset of parameters of a certain statistical model. Under this method, the likelihood is written as a function of one or a few parameters of interest by treating the rest as nuisance parameters. The likelihood is then maximized over the nuisance parameters. To facilitate the construction of the profile-likelihood confidence interval for the parameter of interest, the concept of maximum relative likelihood is employed. The maximum relative likelihood function of the parameter of interest is obtained by maximizing the relative likelihood function of all the parameters in the model over the nuisance parameter, with the value of the 
parameter of interest fixed.

In this paper, we consider approximate confidence intervals for the 2- parameter Weibull distribution based on small type- 2 censored samples using profile-likelihood method. The efficiency of this technique in carrying out interval estimation for the 2-parameter Weibull distribution based on type-2 censored data is then compared with the Wald technique based on the confidence lengths and coverage probabilities.

The remaining part of the paper is organized as follows: materials and methods are presented in Sections 2, 3 and 4. Section 5 presents interval estimate and coverage probability results. In Section 6, discussion of the results is presented. Conclusions are presented in Section 7.

\section{The 2-Parameter Weibull Distribution}

A continuous random variable $X$ is said to have a 2-parameter Weibull distribution if its probability density function is given by

$$
f(x)=\alpha \beta x^{\beta-1} \mathrm{e}^{-\alpha x^{\beta}}, x>0 ; x>0, \alpha>0, \beta>0
$$

where $\alpha$ and $\beta$ are the scale and shape parameters, respectively. This form of the Weibull distribution is known as the 2-parameter Weibull distribution.

The cumulative density function for the 2-parameter Weibull distribution can be derived from Equation (1) and expressed as

$$
F(x)=1-\mathrm{e}^{-\alpha x^{\beta}}, x>0, \alpha>0, \beta>0
$$

Thus, the Weibull reliability at time $x$, which is $1-F(x)=R(x)$, is defined as

$$
R(x)=1-F(x)=\mathrm{e}^{-\alpha x^{\beta}}
$$

The hazard function (sometimes called the failure rate) function, denoted by $h(x)$, of the 2-parameter Weibull distribution is given as

$$
h(x)=\frac{f(x)}{1-F(x)}=\alpha \beta x^{\beta-1} .
$$

Integrating both sides of Equation (4) yields

$$
\ln (1-F(x))=-\int_{0}^{x} h(t) \mathrm{d} t
$$

or

$$
F(x)=1-\mathrm{e}^{-\int_{0}^{x} h(t) \mathrm{d} t}=1-\mathrm{e}^{-H(x)}
$$

where $H(x)=\int_{0}^{x} h(t) \mathrm{d} t=\alpha x^{\beta}$ is called the cumulative hazard function. Also, in terms of the cumulative hazard function the reliability function in Equation (3) can be given as

$$
R(x)=\mathrm{e}^{-H(x)} .
$$

\section{Maximum Likelihood Estimation (MLE) Method}

In this paper, MLE estimates of the 2-parameter Weibull distribution are ob- 
tained using Panahi [3] procedure.

Let $X_{1}<X_{2}<\cdots<X_{r}$ be a type-2 censored sample of size $r$ acquired from a life test on $n$ items whose lifetimes have Weibull. The likelihood function can be written as

$$
L(\alpha, \beta)=\frac{n !}{(n-r) !}\left[\prod_{i=1}^{r} f\left(x_{i}\right)\right]\left[1-F\left(x_{r}\right)\right]^{n-r}
$$

Specifically,

$$
\begin{aligned}
L(\alpha, \beta) & =\frac{n !}{(n-r) !}\left[\prod_{i=1}^{r} \alpha \beta x_{i}^{\beta-1} \mathrm{e}^{-\alpha x_{i}^{\beta}}\right]\left[1-\left(1-\mathrm{e}^{-\alpha x_{r}^{\beta}}\right)\right]^{n-r} \\
& =\frac{n !}{(n-r) !}(\alpha \beta)^{r}\left(\prod_{i=1}^{r} \mathrm{e}^{-\alpha x_{i}^{\beta}}\right)\left(\prod_{i=1}^{r} x_{i}^{\beta-1}\right)\left[\mathrm{e}^{-\alpha x_{r}^{\beta}}\right]^{n-r}
\end{aligned}
$$

Then, the log-likelihood function is written as

$$
\begin{aligned}
\ell(\alpha, \beta)= & \log n !-\log (n-r) !+r \log \alpha+r \log \beta-(n-r) \alpha x_{r}^{\beta} \\
& -\alpha \sum_{i=1}^{r} x_{i}^{\beta}+(\beta-1) \sum_{i=1}^{r} \log x_{i}
\end{aligned}
$$

The MLE of $\alpha$ and $\beta$ denoted as $\hat{\alpha}$ and $\hat{\beta}$ are solutions of the following equations;

$$
\frac{\partial \ell}{\partial \alpha}=\frac{r}{\alpha}-(n-r) x_{r}^{\beta}-\sum_{i=1}^{r} x_{i}^{\beta}=0
$$

and,

$$
\frac{\partial \ell}{\partial \beta}=\frac{r}{\beta}-(n-r) \alpha x_{r}^{\beta} \log x_{r}-\alpha \sum_{i=1}^{r} x_{i}^{\beta} \log x_{i}+\sum_{i=1}^{r} \log x_{i}=0
$$

From Equation (8), the MLE of $\alpha$ is a function of $\beta$ i.e.,

$$
\hat{\alpha}=\frac{r}{(n-r) x_{r}^{\beta}+\sum_{i=1}^{r} x_{i}^{\beta}}
$$

The MLE of $\hat{\beta}$ can be obtained as the solution of

$$
\begin{aligned}
& \left(\frac{r(n-r)}{(n-r) x_{r}^{\beta}+\sum_{i=1}^{r} x_{i}^{\beta}}\right) x_{r}^{\beta} \log x_{r} \\
& +\left(\frac{r}{(n-r) x_{r}^{\beta}+\sum_{i=1}^{r} x_{i}^{\beta}}\right) \sum_{i=1}^{r} x_{i}^{\beta} \log x_{i}-\frac{r}{\beta}-\sum_{i=1}^{r} \log x_{i}=0
\end{aligned}
$$

$\hat{\beta}$ will be obtained numerically via the Newton-Raphson iterative procedure since Equation (11) cannot be solved analytically. Therefore,

$$
\hat{\beta}_{i+1}=\hat{\beta}_{i}+\frac{\left\{\frac{r}{\hat{\beta}_{i}}+S_{1 f}-S_{3 f}-\frac{r}{S_{2 f+} S_{2 s}} S_{3 f}+S_{3 s}\right\}}{\left\{\frac{r}{\hat{\beta}}+S_{4 s}-\frac{r}{\left(S_{2 f}+S_{2 s}\right)^{2}}\left(S_{3 f}+S_{3 s}\right)^{2+\frac{r}{S_{2 f}+S_{2 s}}}\left(S_{4 f}+S_{4 s}\right)\right\}}
$$

where $S_{1 f}=\sum_{i=1}^{r} \log x_{i}, \quad S_{2 f}=\sum x_{i}^{\beta}, \quad S_{2 s}=(n-r) x_{r}^{\beta}, \quad S_{3 f}=\sum_{i=0}^{r} x_{i}^{\beta} \log x_{i}$, 
$S_{3 s}=(n-r) x_{r}^{\beta} \log x_{r}, \quad S_{4 f}=\sum_{i=0}^{r} x_{i}^{\beta}\left(\log x_{i}\right)^{2}$, and $S_{4 s}=(n-r) x_{r}^{\beta}\left(\log x_{r}\right)^{2}$

Once $\hat{\beta}$ has been obtained, $\hat{\alpha}$ is computed as follows:

$$
\hat{\alpha}=\frac{r}{S_{2 f}+S_{2 s}}
$$

\section{Interval Estimation}

Approximate confidence intervals for the parameters of the 2-parameter Weibull distribution are obtained using the Wald technique and profile-likelihood method.

\subsection{Wald Technique}

Approximate Wald confidence intervals for the parameters, $\alpha$ and $\beta$, of the 2-parameter Weibull distribution under the type-2 censoring scheme are also obtained using the steps described in Panahi [3].

Step 1: MLE of $\alpha$ and $\beta$ are obtained as described in Section 3 above.

Step 2: Computation of the observed Fisher information matrix for the MLE's from Equations (8) and (9),

$$
\begin{gathered}
\frac{\partial^{2} \ell}{\partial \alpha^{2}}=-\frac{r}{\alpha^{2}} \\
\frac{\partial^{2} \ell}{\partial \beta^{2}}=-\frac{r}{\beta^{2}}-(n-r) \alpha x_{r}^{\beta}\left(\log x_{r}\right)^{2}-\alpha \sum_{i=1}^{r} x_{i}^{\beta}\left(\log x_{i}\right)^{2}
\end{gathered}
$$

and

$$
\frac{\partial^{2} \ell}{\partial \alpha \partial \beta}=\frac{\partial^{2} \ell}{\partial \beta \partial \alpha}=-(n-r) x_{r}^{\beta} \log x_{r}-\sum_{i=1}^{r} x_{i}^{\beta} \log x_{i}
$$

Equations (14), (15), and (16) will facilitate the computation of the observed Fisher information matrix denoted by

$$
I(\hat{\alpha}, \hat{\beta})=E\left[\begin{array}{cc}
\frac{-\partial^{2} \ell}{\partial \beta^{2}} & \frac{-\partial^{2} \ell}{\partial \beta \partial \alpha} \\
\frac{-\partial^{2} \ell}{\partial \alpha \partial \beta} & \frac{-\partial^{2} \ell}{\partial \alpha^{2}}
\end{array}\right]
$$

The observed Fisher information matrix will aid in the construction of approximate confidence intervals for the parameters based on the limiting normal distribution.

Step 3: Obtaining the inverse of the observed Fisher information matrix in order to find a local estimate of the asymptotic variance-covariance matrix of the MLE. The inverse of the Fisher information matrix is obtained as,

$$
[I(\hat{\alpha}, \hat{\beta})]^{-1}=\left[\begin{array}{cc}
\hat{\sigma}^{2}(\hat{\beta}) & \hat{\sigma}(\hat{\beta}, \hat{\alpha}) \\
\hat{\sigma}(\hat{\alpha}, \hat{\beta}) & \hat{\sigma}^{2}(\hat{\alpha})
\end{array}\right]
$$

Step 4: The Wald confidence intervals. In accordance with the asymptotic 


$$
\begin{aligned}
& \text { theory of MLE, the sampling distribution of } \frac{(\hat{\beta}-\beta)}{\sqrt{\hat{\sigma}^{2}(\hat{\beta})}} \text { can be approximated by } \\
& \text { a standard normal distribution. A two sided } 100(1-\psi) \% \text { normal approximate } \\
& \hat{\beta} \pm Z_{1-\frac{\psi}{2}} \sqrt{\hat{\sigma}^{2}(\hat{\beta})} \\
& \hat{\alpha} \pm Z_{1-\frac{\psi}{2}} \sqrt{\hat{\sigma}^{2}(\hat{\alpha})} \\
& R(\alpha, \beta)=\frac{L(\alpha, \beta)}{L(\hat{\alpha}, \hat{\beta})}=\frac{\log n !-\log (n-r) !+r \log \alpha+r \log \beta-(n-r) \alpha x_{r}^{\beta}-\alpha \sum_{i=1}^{r} x_{i}^{\beta}+(\beta-1) \sum_{i=1}^{r} \log x_{i}}{\log n !-\log (n-r) !+r \log \hat{\alpha}+r \log \hat{\beta}-(n-r) \hat{\alpha} x_{r}^{\hat{\beta}}-\alpha \sum_{i=1}^{r} x_{i}^{\hat{\beta}}+(\hat{\beta}-1) \sum_{i=1}^{r} \log x_{i}} \\
& L_{p}(\beta)=\max _{\alpha} L(\alpha, \beta)=L(\hat{\alpha}(\beta), \beta) \\
& R_{p}(\beta)=\frac{L(\hat{\alpha}(\beta), \beta)}{L(\hat{\alpha}, \hat{\beta})}=R(\hat{\alpha}(\beta), \beta) \\
& R_{p}(\alpha)=\frac{L(\alpha, \hat{\beta}(\alpha))}{L(\hat{\alpha}, \hat{\beta})}=R(\alpha, \hat{\beta}(\alpha)) \\
& r_{p}(\beta)-\log \psi=0 \text {, } \\
& r_{p}(\beta)-\log (0.147)=0 \text {, }
\end{aligned}
$$

\subsection{Profile-Likelihood Technique}


which is solved numerically using the bisection method.

Similarly, the $100 \% \%$ profile-likelihood confidence interval or region for the parameter $\alpha$ is the set of all values for which $R_{p}(\alpha) \geq \psi$ and the end points of this interval are obtained as the solution to the equation

$$
\begin{gathered}
r_{p}(\alpha)-\log \psi=0 \\
r_{p}(\alpha)-\log (0.147)=0
\end{gathered}
$$

which is solved numerically using the bisection method.

The profile-likelihood confidence intervals are fully conditioned on the shape of the profile-likelihood function and in this sense they are optimal. The 14.7\% profile-likelihood interval corresponds approximately to $95 \%$ confidence interval [20].

\section{Simulation Study and Real Data Application}

\subsection{Simulation}

In this study, $n$ identical items were subjected to a life test. The first $r$ items to fail were used for analysis while the remaining $(n-r)$ items were right censored. Time taken for the items to fail was ordered as $X_{1}, \cdots, X_{r}$. The 2- paramater Weibull distribution given by Equation (1) was used to fit the type- 2 censored data.

The simulation of type-2 censored data for the 2-parameter Weibull distribution used the algorithm by Newby [21]. In this approach, the following steps were followed:

Step 1: Set $h_{j}=0$.

Step 2: Generate random numbers $u_{j} \sim \operatorname{Uniform}(0,1), \quad j=0,1, \cdots,(r-1)$.

Step 3: Set $h_{j+1}=h_{j}-\frac{\log \left(u_{j}\right)}{n-j}, j=0,1, \cdots,(r-1)$.

Step 4: $X_{j+1}=H^{-1}\left(h_{j+1}\right), \quad j=0,1, \cdots, r-1$.

One thousand samples each of size $n$ were generated from 2-parameter Weibull distribution by repeated simulations and censored using the type- 2 censoring scheme. This repeated sampling was used to compute coverage probability for the $95 \%$ Wald confidence intervals and $95 \%$ approximate profile-confidence intervals for the parameters of the 2-parameter Weibull distribution for different fixed values of $r, n, \alpha$ and with the value of shape parameter $\beta$ fixed at 1.5. Subroutines to obtain the approximate Wald confidence intervals and the approximate profile-likelihood confidence intervals for the two parameters were developed in the $R$ program (Version 3.5.1). To check the efficiencies of the interval estimates computed by the two techniques, confidence lengths and coverage probabilities for the interval estimates are compared.

\subsection{Simulated Results}

The $95 \%$ Wald confidence intervals and the $95 \%$ profile-likelihood confidence 
approximate intervals for the parameters of the 2- parameter Weibull distribution under type-2 censoring scheme are given in Tables 1-4.

Table 1. Wald confidence intervals, for $n=20$.

\begin{tabular}{|c|c|c|c|c|c|}
\hline$\alpha$ & $r$ & {$\left[\hat{\alpha}_{l}, \hat{\alpha}_{u}\right]$} & CL for $\alpha$ & {$\left[\hat{\beta}_{l}, \hat{\beta}_{u}\right]$} & CL for $\beta$ \\
\hline \multirow{2}{*}{0.5} & 8 & {$[0.19764,1.22446]$} & 1.02682 & {$[0.39738,2.08385]$} & 1.68647 \\
\hline & 4 & {$[0.00000,0.82520$} & 0.82520 & {$[0.038015,2.06786]$} & 2.02985 \\
\hline \multirow{2}{*}{1.0} & 8 & {$[0.22690,1.97821]$} & 1.75131 & {$[0.377045,1.74620]$} & 1.36916 \\
\hline & 4 & {$[0.0000,1.39428]$} & 1.39428 & {$[0.066103,1.93431]$} & 1.86821 \\
\hline \multirow{2}{*}{1.5} & 8 & {$[0.22094,2.54861]$} & 2.32767 & {$[0.37266,1.60855]$} & 1.23589 \\
\hline & 4 & {$[0.00000,1.86983]$} & 1.86983 & {$[0.084172,1.86530]$} & 1.78113 \\
\hline
\end{tabular}

Table 2. Wald confidence intervals, for $n=40$.

\begin{tabular}{cccccc}
\hline$\alpha$ & $\boldsymbol{r}$ & {$\left[\hat{\alpha}_{l}, \hat{\alpha}_{u}\right]$} & CL for $\alpha$ & {$\left[\hat{\beta}_{l}, \hat{\beta}_{u}\right]$} & CL for $\beta$ \\
\hline 0.5 & 8 & {$[0.043312,1.33135]$} & 1.28804 & {$[0.45263,2.01939]$} & 1.56676 \\
& 4 & {$[0.00000,0.86937]$} & 0.86937 & {$[0.063159,2.10891]$} & 2.04575 \\
& 8 & {$[0.00000,2.19627]$} & 2.19627 & {$[0.46025,1.81479]$} & 1.35454 \\
& 4 & {$[0.00000,1.5250]$} & 1.5250 & {$[0.092796,2.01836]$} & 1.92556 \\
& 8 & {$[0.00000,2.88451]$} & 2.88451 & {$[0.46492,1.72284]$} & 1.25792 \\
& 4 & {$[0.00000,2.08531]$} & 2.08531 & {$[0.11064,1.96890]$} & 1.85826 \\
\hline
\end{tabular}

Table 3. The 95\% profile-likelihood approximate confidence intervals, for $n=20$.

\begin{tabular}{|c|c|c|c|c|c|}
\hline$\alpha$ & $r$ & {$\left[\hat{\alpha}_{l}, \hat{\alpha}_{u}\right]$} & CL for $\alpha$ & {$\left[\hat{\beta}_{l}, \hat{\beta}_{u}\right]$} & CL for $\beta$ \\
\hline \multirow{2}{*}{0.5} & 8 & {$[0.32520,1.32290]$} & 0.99770 & {$[0.58814,2.21117]$} & 1.52356 \\
\hline & 4 & {$[0.12235,0.913881]$} & 0.79153 & {$[0.36406,2.17766]$} & 1.81360 \\
\hline \multirow{2}{*}{1.0} & 8 & {$[0.50424,2.05129]$} & 1.54705 & {$[0.55455,1.75009]$} & 1.19554 \\
\hline & 4 & {$[0.18933,1.41421]$} & 1.22488 & {$[0.41767,1.84438]$} & 1.42671 \\
\hline \multirow{2}{*}{1.5} & 8 & {$[0.63331,2.57639]$} & 1.94308 & {$[0.54927,1.57123]$} & 1.02196 \\
\hline & 4 & {$[0.24103,1.80033]$} & 1.5593 & {$[0.45431,1.70303]$} & 1.24872 \\
\hline
\end{tabular}

Table 4. The 95\% profile-likelihood approximate confidence intervals, for $n=40$.

\begin{tabular}{cccccc}
\hline$\alpha$ & $\boldsymbol{r}$ & {$\left[\hat{\alpha}_{l}, \hat{\alpha}_{u}\right]$} & CL for $\alpha$ & {$\left[\hat{\beta}_{l}, \hat{\beta}_{u}\right]$} & CL for $\beta$ \\
\hline 0.5 & 8 & {$[0.31436,1.27880]$} & 0.96444 & {$[0.72555,1.88895]$} & 1.16340 \\
& 4 & {$[0.10992,0.82101]$} & 0.71109 & {$[0.50643,1.89330]$} & 1.38687 \\
& 8 & {$[0.48605,1.97731]$} & 1.49126 & {$[0.74387,1.63058]$} & 0.88671 \\
& 4 & {$[0.17309,1.29293]$} & 1.11984 & {$[0.58740,1.68967]$} & 1.10227 \\
& 8 & {$[0.61742,2.51172]$} & 1.8943 & {$[0.74968,1.52322]$} & 0.77354 \\
\hline
\end{tabular}


From the simulation results in the Table 1, it can be observed that for a fixed value of the scale parameter $\alpha$ and overall sample size $n$, as the effective sample size $r$ increases, the length of the Wald confidence interval for the shape parameter $\beta$ decreases. On the other hand, it can be observed that for a fixed value of the scale parameter $\alpha$ and overall sample size $n$, as the effective sample size $r$ increases, the length of the Wald confidence interval for the scale parameter $\alpha$ also increases.

From the simulation results in Table 2, it can be observed that for a fixed value of the scale parameter $\alpha$ and overall sample size $n$, as the effective sample size $r$ increases, the length of the Wald confidence interval for the shape parameter $\beta$ decreases. On the other hand, it can be observed that for a fixed value of the scale parameter $\alpha$ and overall sample size $n$, as the effective sample size $r$ increases, the length of the Wald confidence interval for the scale parameter $\alpha$ also increases.

Moreover, it can be observed that when the effective sample size $r=8$, most of the Wald confidence interval results for the shape parameter $\beta$ in Table 2 are narrower than the Wald confidence interval results for the shape parameter $\beta$ in Table 1 . Also, it can be observed that the Wald confidence interval results for the scale parameter $\alpha$ in Table 1 are narrower than those in Table 2 .

From the simulation results in Table 3, it can be observed that for a fixed value of the scale parameter $\alpha$ and overall sample size $n$, as the effective sample size $r$ increases, the length of the approximate Profile-likelihood confidence interval for the shape parameter $\beta$ decreases. On the other hand, it can be observed that for a fixed value of the scale parameter $\alpha$ and overall sample size $n$, as the effective sample size $r$ increases, the length of the approximate profile-likelihood confidence interval for the scale parameter $\alpha$ also increases.

From the simulation results in Table 4, it can be observed that for a fixed value of the scale parameter $\alpha$ and overall sample size $n$, as the effective sample size $r$ increases, the length of the approximate Profile-likelihood confidence interval for the shape parameter $\beta$ decreases. On the other hand, it can be observed that for a fixed value of the scale parameter $\alpha$ and overall sample size $n$, as the effective sample size $r$ increases, the length of the approximate profile-likelihood confidence interval for the scale parameter $\alpha$ also increases.

Furthermore, it can be observed that the 95\% profile-likelihood confidence interval results for the scale parameter $\alpha$ and shape parameter $\beta$ in Table 4 are narrower than the $95 \%$ profile-likelihood confidence interval results in Table 3. That is, the 95\% profile-likelihood confidence intervals for $\beta$ and $\alpha$ become narrower when the sample size $n$ is increased.

Some selected $95 \%$ confidence interval estimates for the shape parameter $\beta$ obtained using the aforementioned techniques are plotted on the relative profilelikelihood function graph of $\beta$ in order to compare their plausibilities. These plots are given below. 

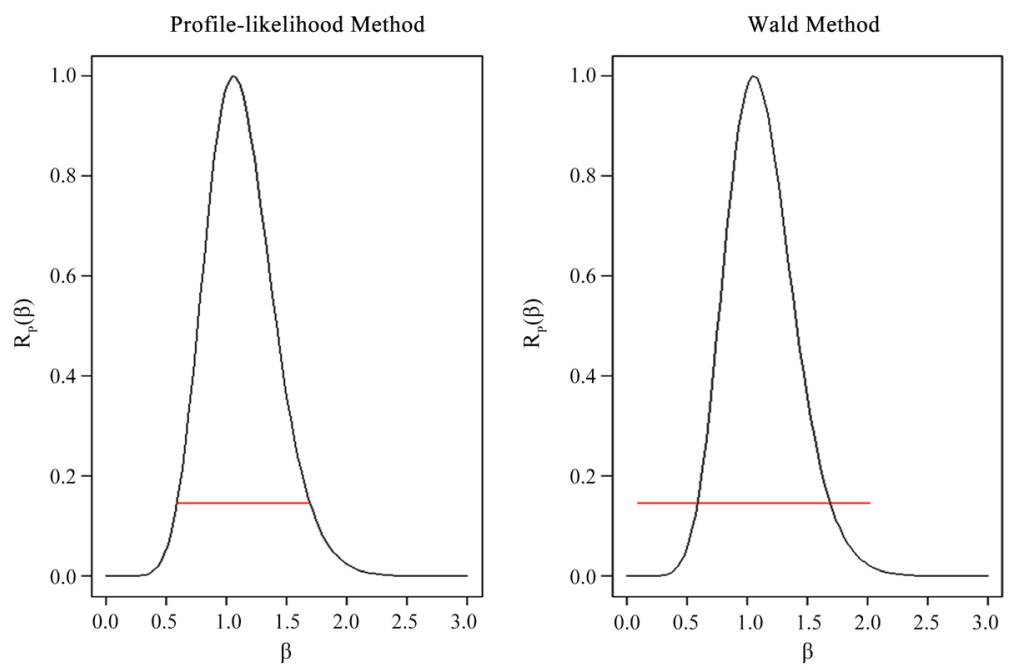

Figure 1. Plots of relative profile-likelihood function of $\beta$ for $\alpha=1, \beta=1.5$, $r=4, n=40$.


Figure 2. Plots of relative profile-likelihood function of $\beta$ for $\alpha=1.5, \beta=1.5$, $r=4, n=40$.


Figure 3. Plots of relative profile-likelihood function of $\beta$ for $\alpha=0.5, \beta=1.5$, $r=8, n=40$. 

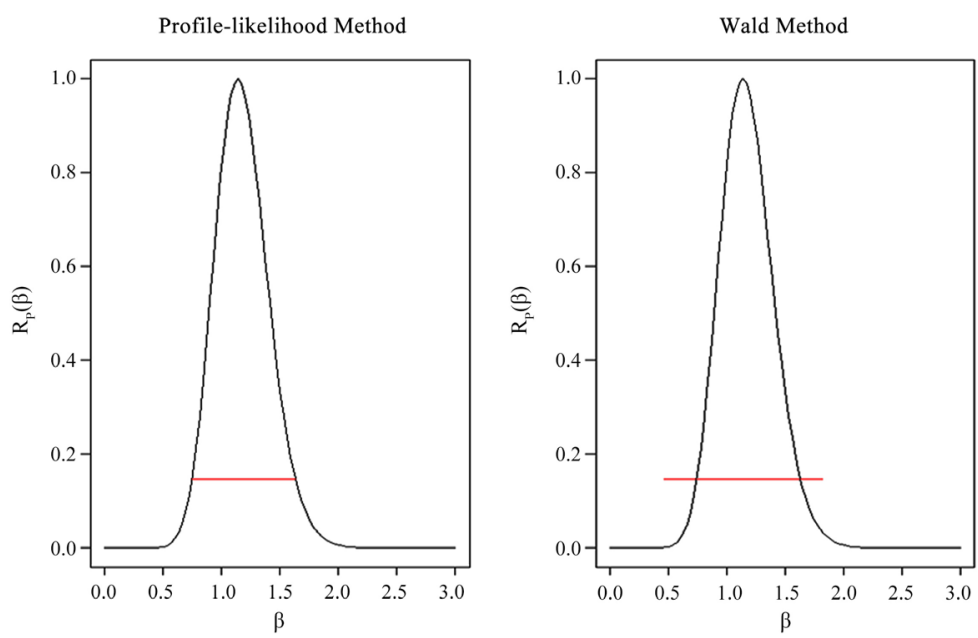

Figure 4. Plots of relative profile-likelihood function of $\beta$ for $\alpha=1, \beta=1.5$, $r=8, n=40$.
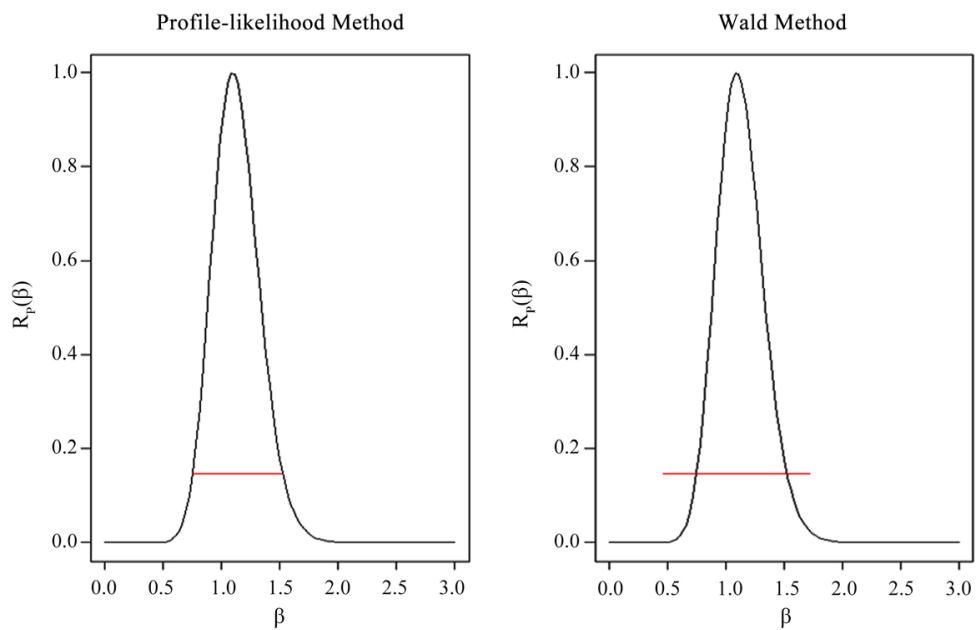

Figure 5. Plots of relative profile-likelihood function of $\beta$ for $\alpha=1.5, \beta=1.5$, $r=8, n=40$.
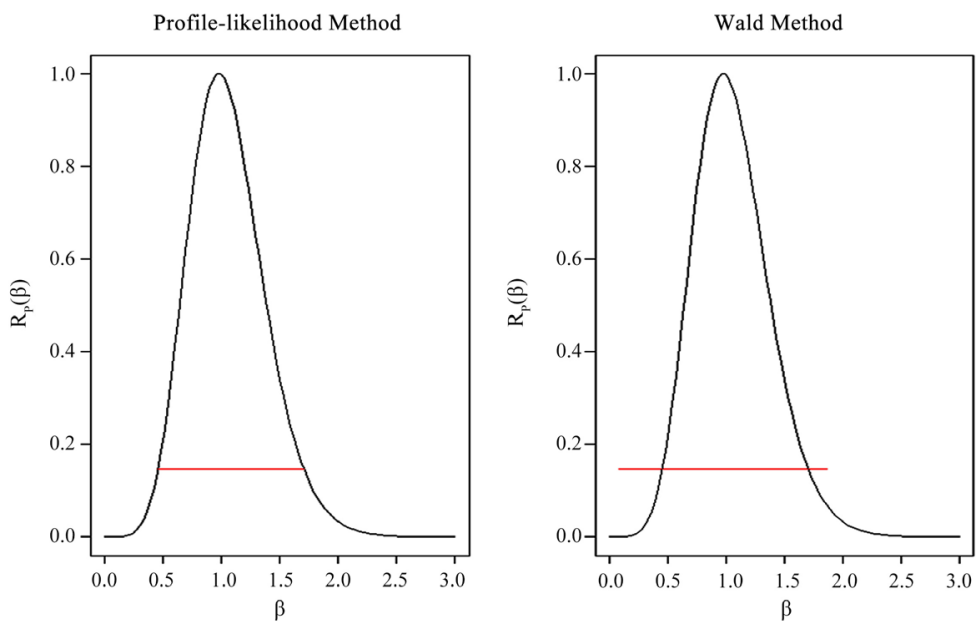

Figure 6. Plots of relative profile-likelihood function of $\beta$ for $\alpha=1.5, \beta=1.5$, $r=4, n=20$. 

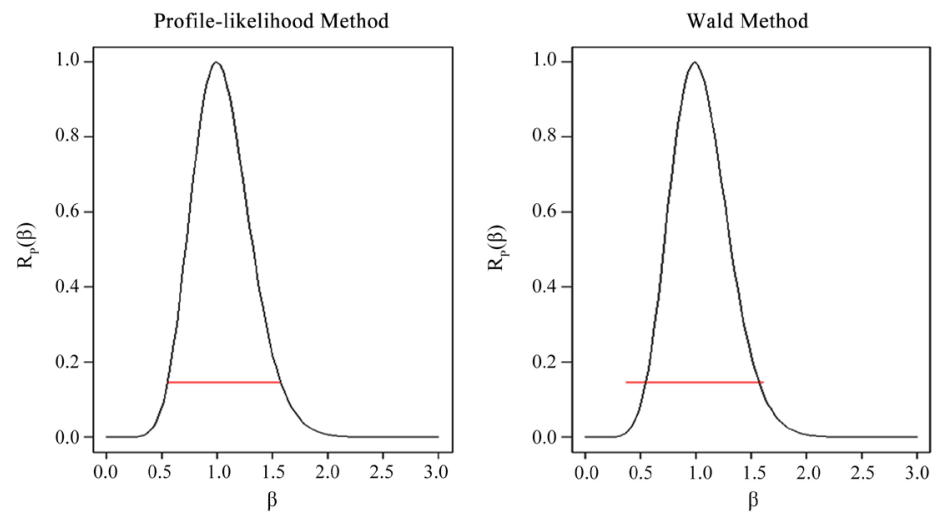

Figure 7. Plots of relative profile-likelihood function of $\beta$ for $\alpha=1.5, \beta=1.5, r=8, n=20$.

It is evident from Figures 1-7 that the length of the interval estimates for the shape parameter $\beta$ associated with the profile-likelihood method are narrower as compared to those associated with the Wald technique irrespective of the sample size $n$.

Similarly, a subset of interval estimates for the scale parameter $\alpha$ obtained using the aforementioned techniques, are plotted on the profile-likelihood function graph of $\alpha$ in order to illustrate their plausibilities. The horizontal red line denotes $14.7 \%$ percentile which is equivalent to $95 \%$ confidence interval. These plots are given below.


Figure 8. Plots of relative profile-likelihood function of $\alpha$ for $\alpha=1, \beta=1.5, r=4, n=40$.
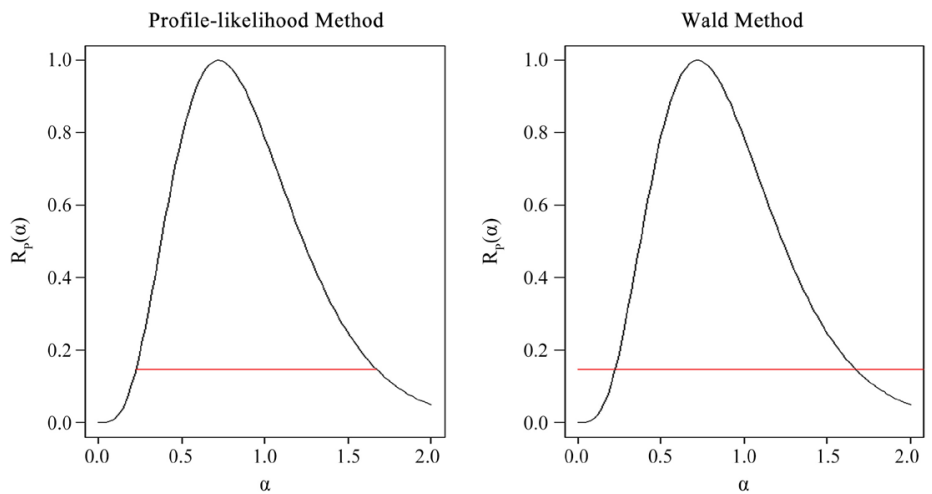

Figure 9. Plots of relative profile-likelihood function of $\alpha$ for $\alpha=1.5, \beta=1.5, r=4, n=40$. 

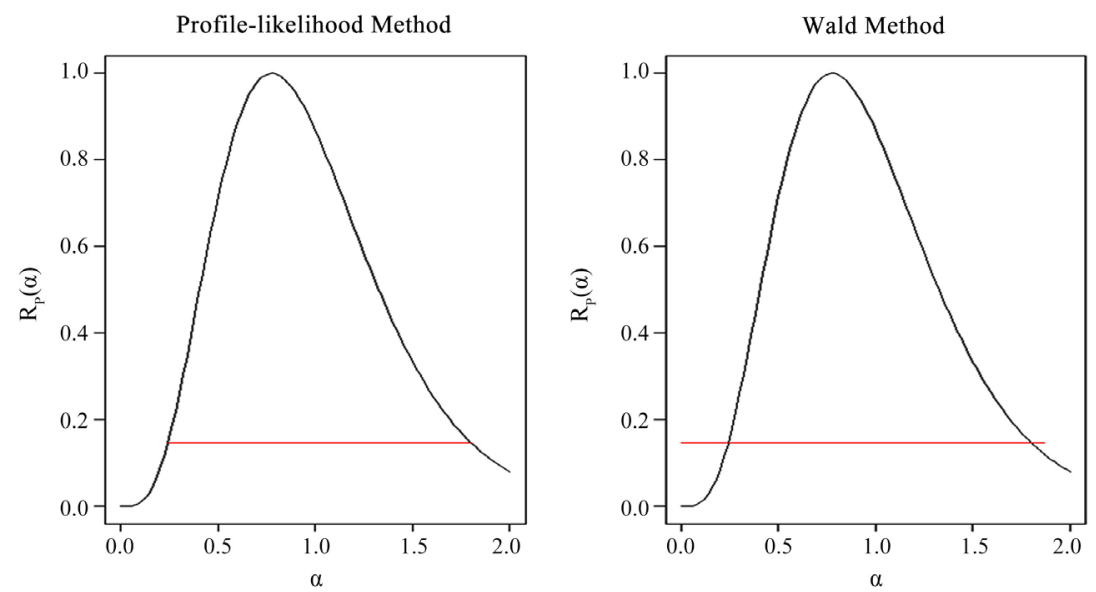

Figure 10. Plots of relative profile-likelihood function of $\alpha$ for $\alpha=1.5, \beta=1.5, r=4$, $n=20$.

It is evident from Figures 8-10 above the length of the interval estimates for the scale parameter $\alpha$ associated with the profile-likelihood method are narrower as compared to those associated with the Wald technique irrespective of the size of the sample size $n$.

\subsection{The Coverage Probabilities Results}

The $95 \%$ coverage probability results for the interval estimates of the 2-parameter Weibull distribution associated with Wald and profile-likelihood technique under type- 2 censoring scheme are given in Tables 5-8.

From the results in Table 5, it can be observed that most of the coverage probabilities for the shape parameter $\beta$ are greater than or relatively close to the nominal coverage probability (0.95). Similarly, it can also be observed that the coverage probabilities of the scale parameter $\alpha$ are less than the nominal coverage probability (0.95).

From the results in Table 6, it can be observed that most of the coverage probabilities for the shape parameter $\beta$ are greater than or relatively close to the nominal coverage probability (0.95). On the other hand, it can be observed that the coverage probabilities for the scale parameter $\alpha$ are less than or relatively close to the nominal coverage probability (0.95).

Table 5. The 95\% wald approximate coverage probabilities, for $n=20$.

\begin{tabular}{cccc}
\hline $\boldsymbol{\alpha}$ & $\boldsymbol{r}$ & $\boldsymbol{C P _ { \alpha }}$ & $\boldsymbol{C P _ { \beta }}$ \\
\hline 0.5 & 8 & 0.845 & 0.956 \\
& 4 & 0.910 & 0.976 \\
1.0 & 8 & 0.798 & 0.912 \\
& 4 & 0.895 & 0.968 \\
1.5 & 8 & 0.682 & 0.858 \\
& 4 & 0.848 & 0.962
\end{tabular}


Table 6. The 95\% wald approximate Coverage probabilities, for $n=40$.

\begin{tabular}{cccc}
\hline $\boldsymbol{\alpha}$ & $\boldsymbol{r}$ & $\boldsymbol{C} \boldsymbol{P}_{\boldsymbol{\alpha}}$ & $\boldsymbol{C \boldsymbol { P } _ { \boldsymbol { \beta } }}$ \\
\hline 0.5 & 8 & 0.915 & 0.958 \\
& 4 & 0.948 & 0.977 \\
1.0 & 8 & 0.844 & 0.942 \\
& 4 & 0.920 & 0.976 \\
1.5 & 8 & 0.767 & 0.918 \\
& 4 & 0.897 & 0.976
\end{tabular}

Table 7. The $95 \%$ profile-likelihood coverage probabilities, for $n=20$.

\begin{tabular}{cccc}
\hline $\boldsymbol{\alpha}$ & $\boldsymbol{r}$ & $\boldsymbol{C} \boldsymbol{P}_{\boldsymbol{\alpha}}$ & $\boldsymbol{C P _ { \beta }}$ \\
\hline 0.5 & 8 & 0.873 & 0.923 \\
& 4 & 0.949 & 0.947 \\
1.0 & 8 & 0.927 & 0.945 \\
& 4 & 0.958 & 0.966 \\
& 8 & 0.682 & 0.881 \\
\hline & 4 & 0.860 & 0.937 \\
\hline
\end{tabular}

Table 8. The 95\% profile-likelihood coverage probabilities, for $n=40$.

\begin{tabular}{cccc}
\hline $\boldsymbol{\alpha}$ & $\boldsymbol{r}$ & $\boldsymbol{C P _ { \alpha }}$ & $\boldsymbol{C P _ { \beta }}$ \\
\hline 0.5 & 8 & 0.955 & 0.961 \\
& 4 & 0.945 & 0.924 \\
1.0 & 8 & 0.886 & 0.889 \\
& 4 & 0.892 & 0.937 \\
1.5 & 8 & 0.674 & 0.817 \\
& 4 & 0.755 & 0.905 \\
\hline
\end{tabular}

Based on the coverage probability results given in Table 5 and Table 6, it can be observed that Wald coverage probabilities improve when sample size $n$ is increased.

From the results given in Table 7, it can be observed that most of the coverage probabilities for the shape parameter $\beta$ are very close to the nominal coverage probability (0.95) and the coverage probabilities for the scale parameter $\alpha$ are less than or relatively close to the nominal coverage probability.

From results given in Table 8 , it can be observed that the coverage probabilities for the shape parameter $\beta$ are either less than, greater than or relatively close to the nominal coverage probability (0.95). Similarly, it can be observed that most of the coverage probabilities for the scale parameter $\alpha$ are less than the nominal coverage probability.

Moreover, based on the coverage probability simulated results in Table 7 and Table 8, it can be observed that most of the profile-likelihood coverage probabilities for both parameters are close to the nominal coverage probability (0.95) 
when the sample size $n$ is small, that is, when $n=20$.

\subsection{Real Data Analysis}

For illustrative purposes, real type-2 censored life data given in Nelson [22] is deployed to construct the approximate Wald confidence intervals and the approximate profile-likelihood confidence intervals for parameters of the 2- parameter Weibull distribution. The data represents the lifetimes in hours of twelve appliance cords subjected to a flex test and the test terminated after the failure of the first nine appliance cords.

The data is as given below.

$$
\text { 57.5, 77.8, 88.0, 98.4, 102.1, 105.3, 139.3, 143.9, } 148.0
$$

Since the empirical hazard function exhibits an increasing trend, the 2- parameter Weibull distribution can be used for analyzing the above real type- 2 censored life data as described by Kundu and Raqab [23]. For the sake of computational ease, we have divided all the data points by 100 .

Using the above real data, the MLE's of the parameters $\alpha$ and $\beta$ of the 2-parameter Weibull distribution under type-2 censoring scheme are obtained as 0.3392821 and 4.4127589 respectively. Deploying the Wald method, the $95 \%$ approximate confidence intervals for $\alpha$ and $\beta$ are obtained as [0.0485729, 0.629991] and $[2.195963,6.629554]$ respectively. Further, using the profile-likelihood technique, the $95 \%$ approximate confidence intervals for $\alpha$ and $\beta$ are obtained as $[0.1631579,0.6113762]$ and $[2.724605,6.025074]$, respectively. Based on these results, it can be observed that the interval estimates obtained by the profile-likelihood method are narrower as compared to those obtained using the Wald technique. The interval estimates obtained using both approaches are plotted on the relative profile-likelihood function graphs of $\alpha$ and $\beta$ in order to illustrate their plausibilities. These plots are given in Figures 11-12.

It is evident from Figure 11 and Figure 12 that the interval estimates obtained by the profile-likelihood method are narrower as compared to those obtained using the Wald technique.
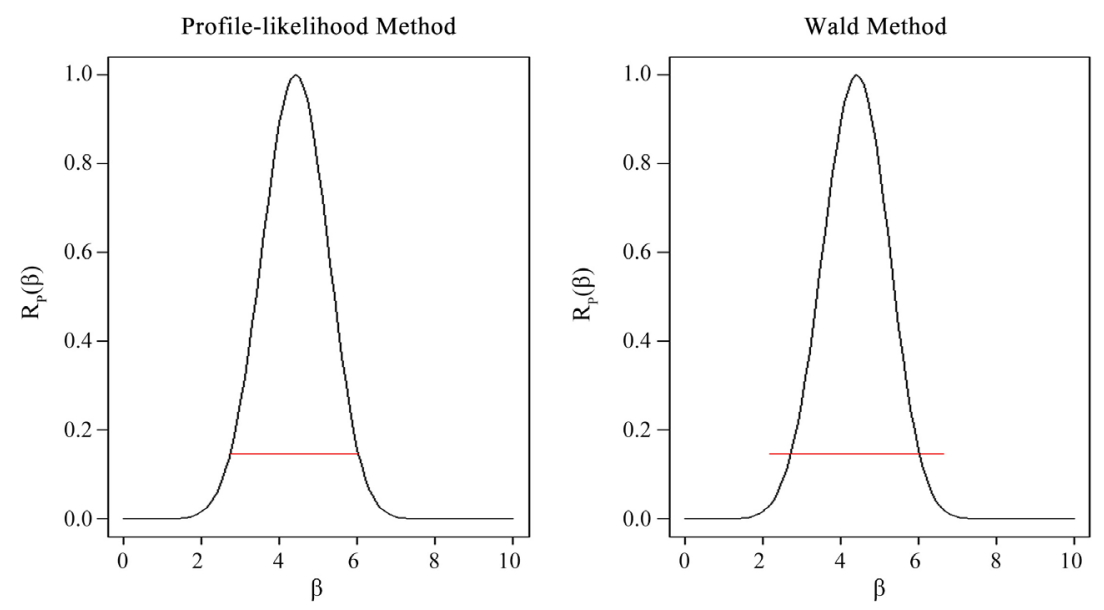

Figure 11. Plots of relative profile-likelihood function of $\beta$ for $=9, n=12$. 

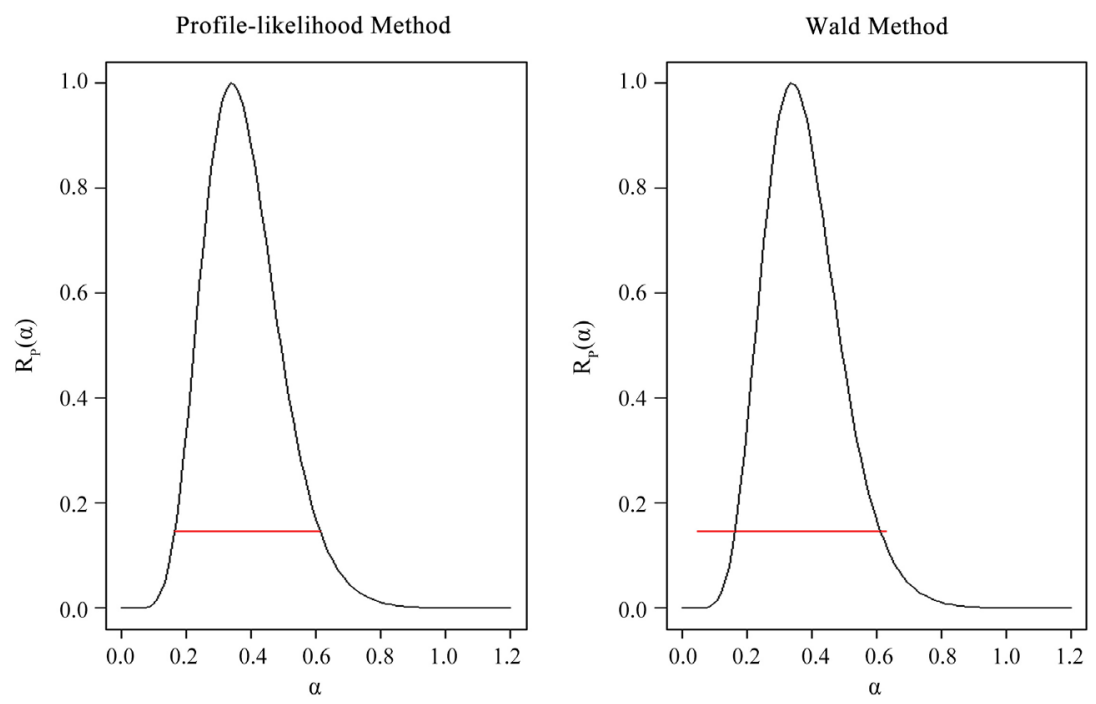

Figure 12. Plots of relative profile-likelihood function of $\alpha$ for $=9, n=12$.

\section{Discussion}

In this paper, we have considered interval estimation of a 2-parameter Weibull distribution based on type- 2 censored data. We have used profile-likelihood technique to compute approximate confidence intervals for the parameters of the Weibull distribution under different values of sample size $n$, different values of effective sample size $r$ and different values of the scale parameter $\alpha$ with value of the shape parameter $\beta$ fixed as 1.5. From simulation results in Table 1 and Table 2, it can be observed that the confidence lengths of interval estimates for $\alpha$ and $\beta$ obtained by Wald technique are relatively wide for both small and large sample size.

From the simulation results in Table 3 and Table 4 it can be observed the confidence lengths of interval estimates for the scale parameter $\alpha$ and shape parameter $\beta$ obtained by profile-likelihood technique are relatively narrow for both small and large sample size. The profile-likelihood technique produced improved confidence interval estimates for $\alpha$ and $\beta$ given in Table 3 to Table 4 which are relatively narrower for both small and large sample size.

From the simulation results in Tables 5-8 it can be observed that, for small sample size $n=20$, most of the coverage probability results for the parameters $a$ and $\beta$ associated with the Wald method are not very stable because they are below the nominal coverage probability $(0.95)$. On the other hand, it can be observed that for large sample size $n=40$, most of the coverage probability results for the parameters $\alpha$ and $\beta$ associated with the Wald method are greater than or relatively close to the nominal coverage probability (0.95). Moreover, the coverage probability results for the parameters $\alpha$ and $\beta$ associated with the profile-likelihood technique are not that much stable when the sample size $n$ is large because most of them are less than the nominal coverage probability but when the sample size $n$ is small, most of them are relatively close to the nominal coverage probability. 
In interval estimation, profile-likelihood technique is preferred over Wald method in the context of small samples because it yields more plausible interval estimates. The confidence lengths in the case of the profile-likelihood method are narrower as compared to those of the Wald technique. This can be explained by the fact that Wald method is appropriate for large samples [24]. Therefore, it may give inaccurate interval estimates when the sample size is small.

The Profile-likelihood interval estimates obtained in this paper can be used to make better inferences in life-testing experiments when the sample size $n$ used is small. This will save time and minimize the cost of performing life-testing experiments.

\section{Conclusion}

Based on the simulation and real data results, it can be concluded that the Profile-likelihood technique outperforms the Wald method because it yields narrower interval estimates. The method applied in this paper can be extended to constructing approximate confidence intervals for the parameters of 2- parameter Weibull distribution based on hybrid censored data.

\section{Conflicts of Interest}

The authors declare no conflicts of interest regarding the publication of this paper.

\section{References}

[1] Park, C. and Lee, S.B. (2012) Parameter Estimation from Censored Samples Using the Expectation-Maximization Algorithm. arXiv preprint arXiv:1203.3880, Clemson University, Clemson.

[2] Støtvig, J.G. (2014) Censored Weibull Distributed Data in Experimental Design. Master's Thesis, Institutt for Matematiske Fag.

[3] Panahi, H. and Asadi, S. (2011) Estimation of the Weibull Distribution Based on Type-II Censored Samples. Applied Mathematical Sciences, 5, 2549-2558.

[4] Cho, Y., Sun, H. and Lee, K. (2014) An Estimation of the Entropy for a Rayleigh Distribution Based on Doubly-Generalised Type-II Hybrid Censored Samples. Entropy, 16, 3655-3669. https://doi.org/10.3390/e16073655

[5] Balakrishnan, N. and Mitra, D. (2013) Likelihood Inference Based on Left truncated and Right Censored Data from a Gamma Distribution. IEEE Transactions on Reliability, 62, 679-688. https://doi.org/10.1109/TR.2013.2273039

[6] Sindhu, T.N., Feroze, N. and Aslam, M. (2016) Study of the Left Censored Data from the Gumbel Type II Distribution under a Bayesian Approach. Journal of Modern Applied Statistical Methods, 15, Article No. 10. https://doi.org/10.22237/jmasm/1478002080

[7] Yenilmez, I., Kantar, Y. M. and Acitas, S. (2018) Estimation of Censored Regression Model in the Case of Non-Normal Error. Sigma Journal of Engineering and Natural Sciences-Sigma Muhendislik ve Fen Bilimleri Dergisi, 36, 513-521.

[8] Emura, T. and Shiu, S. K. (2016) Estimation and Model Selection for Left-Truncated and Right-Censored Lifetime Data with Application to Electric Power Transformers Analysis. Communications in Statistics-Simulation and Computation, 45, 3171- 
3189. https://doi.org/10.1080/03610918.2014.925923

[9] Guure, C.B., Ibrahim, N.A., Dwomoh, D. and Bosomprah, S. (2015) Bayesian Statistical Inference of the Loglogistic Model with Interval-Censored Lifetime Data. Journal of Statistical Computation and Simulation, 85, 1567-1583.

https://doi.org/10.1080/00949655.2014.881813

[10] Khoolenjani, N.B. and Shahsanaie, F. (2016) Estimating the Parameter of Exponential Distribution under Type-II Censoring from Fuzzy Data. Journal of Statistical Theory and Applications, 15, 181-195. https://doi.org/10.2991/jsta.2016.15.2.8

[11] Kim, N. (2014) Estimation for Mean and Standard Deviation of Normal Distribution under Type II Censoring. Communications, 21, 529-538. https://doi.org/10.5351/CSAM.2014.21.6.529

[12] Pradhan, B. and Kundu, D. (2014) Analysis of Interval-Censored Data with Weibull Lifetime Distribution. Sankhya B, 76, 120-139.

https://doi.org/10.1007/s13571-013-0076-1

[13] Ferreira, L.A. and Silva, J.L. (2017) Parameter Estimation for Weibull Distribution with Right Censored Data Using EM Algorithm. Eksploatacja i NiezawodnośćMaintenance and Reliability, 19, 310-315. http://dx.doi.org/10.17531/ein.2017.2.20

[14] Ateya, S.F. and Alharthi, A.S. (2014) Estimation under a Finite Mixture of Modified Weibull Distributions Based on Censored Data via EM Algorithm with Application. Journal of Statistical Theory and Applications, 13, 196-204. https://doi.org/10.2991/jsta.2014.13.3.1

[15] Nelson, W. (1990) Accelerated Testing. John Willey \& Sons, Inc., Hoboken, 60-65.

[16] Karakoca, A. and Pekgör, A. (2019) Maximum Likelihood Estimation of the Parameters of Progressively Type-2 Censored Samples from Weibull Distribution Using Genetic Algorithm. Academic Platform Journal of Engineering and Science, 7, 189-199. https://doi.org/10.21541/apjes.452564

[17] Sirvanci, M. and Yang, G. (1984) Estimation of the Weibull Parameters under Type I Censoring. Journal of the American Statistical Association, 79, 183-187. https://doi.org/10.1080/01621459.1984.10477082

[18] Engeman, R.M. and Keefe, T.J. (1982) On Generalized Least Squares Estimation of the Weibull Distribution. Communications in Statistics-Theory and Methods, 11, 2181-2193. https://doi.org/10.1080/03610928208828380

[19] Jeng, S.-L. and Meeker, W. (2000) Comparisons of Approximate Confidence Interval Procedures for Type I Censored Data. Technometrics, 42, 135-148. https://doi.org/10.1080/00401706.2000.10485992

[20] Kalbfleisch, J.G. (1985) Probability and Statistical Inference. Springer-Verlag Inc., New York

[21] Newby, M.J. (1979) The Simulation of Order Statistics from Life Distributions. Journal of the Royal Statistical Society. Series C (Applied Statistics), 28, 298-300. https://doi.org/10.2307/2347202

[22] Nelson, W. (1982) Applied Life Data Analysis. John Wiley \& Sons, New York. https://doi.org/10.1002/0471725234

[23] Kundu, D. and Raqab, M. Z. (2012) Bayesian Inference and Prediction of Order Statistics for a Type-II Censored Weibull Distribution. Journal of Statistical Planning \& Inference, 142, 41-47. https://doi.org/10.1016/j.jspi.2011.06.019

[24] Kariuki, V.W., Orawo, L.A. and Islam, A.S. (2014) Likelihood Based Estimation of the Parameters of a Log-Linear Nonhomogeneous Poisson Process. International Journal of Science and Research, 3, 200-204. 